\title{
Blocks and progressions in subset sum sets
}

\author{
by \\ Vsevolod F. Lev (Haifa)
}

0. The summary. Let $A$ be a finite set of positive integers and let $A^{*}$ be the set of all subset sums of $A$. We show that if $A$ is dense enough (say, $A \subseteq[1, l]$ and $\left.|A| \geq 5(l \ln l)^{1 / 2}\right)$, then $A^{*}$ contains a long block of consecutive integers or at least a long homogeneous arithmetic progression. This refines earlier results due to Freiman and Sárközy.

1. The background. Most problems in additive number theory share, with some deviations, the same common pattern: given a set $A$ of positive integers, show that the set of all numbers representable as a sum of elements of $A$ is "large". The exact meaning of "large" can vary: one may actually count the numbers representable, or may wish to prove that there are, say, perfect squares, or prime numbers among them. Here we are concerned with the situation when $A$ is finite, the summands are pairwise distinct, and any number of summands is allowed. (For the case of $A$ infinite see Appendix; for representations with repetitions see [S89, Le97]; for representations with a fixed number of summands see Section 4.)

We let

$$
A^{*}:=\left\{a_{1}+\ldots+a_{k}: a_{1}<\ldots<a_{k}, a_{1}, \ldots, a_{k} \in A\right\},
$$

the set of all integers representable as a sum of a number of pairwise distinct elements of $A$. Plainly, $A^{*} \subseteq[0, \sigma(A)]$, where $\sigma(A)$ is the sum of all elements of $A$; moreover, $A^{*}$ is symmetric about $\sigma(A) / 2$, the midpoint of the interval: $s \in A^{*}$ if and only if $\sigma(A)-s \in A^{*}$. It is the set $A^{*}$ which is to be shown "large".

Perhaps, the most radical approach is to prove that $A^{*}$ contains long blocks of consecutive integers, provided that $A$ is sufficiently dense. It is easily seen, however, that $A^{*}$ may fail to contain such blocks; this happens, for instance, if all or almost all elements of $A$ have a common divisor $d>1$. For this reason, instead of a block of integers one may seek in $A^{*}$ an arith-

2000 Mathematics Subject Classification: Primary 11B05; Secondary 11B13, 11B25, 05D05, 11P99. 
metic progression of the form

$$
\{(m+1) d,(m+2) d, \ldots,(m+M) d\} .
$$

Such progressions are called homogeneous; they are just sets of consecutive multiples of an integer number $d$.

Two results in this direction were established independently and almost simultaneously by Freiman and Sárközy.

Theorem A (Freiman, [Fr93]). There exist absolute positive constants $c_{1}$ and $c_{2}$ with the following property. Let $A \subseteq[1, l]$ be a set of $n=|A|$ integers satisfying $n>c_{1}(l \ln l)^{1 / 2}$. Write $\sigma(A)=\sum_{a \in A} a$. Then there is a positive integer $d \leq 3 l / n$ such that $A^{*}$ contains all multiples of $d$ which fall into the interval $\left[\frac{1}{2} \sigma(A)-c_{2} d n^{2}, \frac{1}{2} \sigma(A)+c_{2} d n^{2}\right]$.

Theorem B (Sárközy, [S94, Theorem 4]). Let $A \subseteq[1, l]$ be a set of $n=|A|$ integers satisfying

$$
l>2500, \quad n>200(l \ln l)^{1 / 2} .
$$

Then there are integers $y, z$, and $d \geq 1$ such that

$$
d<10^{4} \frac{l}{n}, \quad z>7^{-1} 10^{-4} n^{2}, \quad \frac{z}{y}>7^{-1} 10^{-4} \frac{n^{2}}{l},
$$

and $\{y d,(y+1) d, \ldots, z d\} \subseteq A^{*}$.

Both theorems impose similar density restrictions on $A$, both give similar bounds for the difference $d$ and the number of terms of the progression. The minor discrepancy is that Theorem A guarantees that the progression is centered about $\sigma(A) / 2$, while Theorem B provides, instead, a lower bound for the "logarithmic length" $(z / y)$ of the progression.

There are a number of papers where other results of this kind are obtained; we mention [AF88, Li89, C90, EF90, GM91, Le98, C99]. Theorems $\mathrm{A}$ and $\mathrm{B}$, however, are most applicable and strongest up to date in the sense that the assumption $n \gg(l \ln l)^{1 / 2}$ is less restrictive than those made elsewhere. (The only exception is [C99, Corollary 2.9] which is based on Theorem A and inherits its assumption.)

2. The results. We develop Theorems A and B in the following directions.

First, we extend significantly the length of the progression which is guaranteed to exist in $A^{*}$ and show that in fact this progression stretches almost onto the whole interval $[0, \sigma(A)]$.

Second, we formulate an arithmetic condition such that if $A$ satisfies this condition, then the progression in question has difference one and is, therefore, a block of consecutive integers. Our condition is, in a sense, the weakest possible. 
Third, we get rid of unspecified or excessively large constants replacing them by reasonably small ones.

For $y$ and $z$ real, $y \leq z$, we denote by $[y, z]$ the set of all integer numbers between $y$ and $z$. Given a positive integer $q$, by $N_{q}(A)$ we denote the number of elements of $A$ not divisible by $q$.

Theorem 1. Let $A \subseteq[1, l]$ be a set of $n=|A| \geq n_{0}$ integers, where $n_{0}$ is a sufficiently large absolute constant. Suppose that

$$
n \geq 20(l \ln n)^{1 / 2}
$$

and that for any positive integer $q<2 l / n$ we have $N_{q}(A) \geq q-1$. Then

$$
[\lambda \sigma(A),(1-\lambda) \sigma(A)] \subseteq A^{*},
$$

where $\sigma(A)$ is the sum of all elements of $A$ and $\lambda=280 l / n^{2}$.

We postpone the proof of this and other results of this paper (that will be presented shortly) until Sections $4-7$.

REMARKs. 1 . The value of $\lambda$ cannot be replaced by $\lambda_{0}=l /\left(2 n^{2}\right)$ : for any $l$ and $n$ such that $3 n \leq l \leq n^{2} / 6$ there exist an $n$-element set $A \subseteq[1, l]$ satisfying $N_{q}(A) \geq q-1$ for any $q<2 l / n$ and an integer $s \in\left[\lambda_{0} \sigma(A)\right.$, $\left.\left(1-\lambda_{0}\right) \sigma(A)\right]$ such that $s \notin A^{*}$. To see this, take $A=[l-(n-1), l], h=$ $\lfloor l /(n-1)\rfloor-1>l /(2(n-1))>l /(2 n)$ and $s=h l$, so that $\lambda_{0} \sigma(A) \leq$ $s \leq\left(1-\lambda_{0}\right) \sigma(A)$ (as follows by an easy verification). Then $s$ cannot be represented as a sum of $h+1$ or more distinct elements of $A$, for any such sum is greater than $(h+1)(l-(n-1))=s+(l-(h+1)(n-1)) \geq s$; and similarly, $s$ cannot be represented as a sum of $h$ or less distinct elements of $A$.

2. If $A$ contains less than $q-1$ elements not divisible by $q$ for an integer $q \geq 1$, then it can happen that some residue classes modulo $q$ are not represented in $A^{*}$. (For instance, consider the situation when each $a \in A$ is either zero or one modulo q.) Clearly, in this case $A^{*}$ does not contain $q$ consecutive integers.

3. It is easily seen that for any fixed $\varepsilon>0$ and $n$ large enough, the condition $n \geq 20(l \ln n)^{1 / 2}$ follows from $n \geq(10 \sqrt{2}+\varepsilon)(l \ln l)^{1 / 2}$. Thus, we have in effect replaced Freiman's constant $c_{1}$ and Sárközy's constant 200 by the constant $10 \sqrt{2}$. It will be seen later that $10 \sqrt{2}$ can be further reduced to a value smaller than 5 , but this comes at the expense of decreasing the block length.

As a corollary, we show that even if $A$ fails to satisfy $N_{q}(A) \geq q-1$ for all $q<2 l / n$, the subset sum set $A^{*}$ still contains a long homogeneous progression. Let $\sigma(A), \lambda$, and $N_{q}(A)$ be as in Theorem 1 . 
Corollary 1. Let $A \subseteq[1, l]$ be a set of $n=|A| \geq n_{0}$ integers, where $n_{0}$ is a sufficiently large absolute constant. Suppose that

$$
n \geq 20(l \ln n)^{1 / 2} \text {. }
$$

Then there exists a positive integer $d<2 l / n$ such that $A^{*}$ contains all multiples of $d$ that fall into the interval

$$
I_{A}:=[\lambda \sigma(A),(1-\lambda) \sigma(A)] .
$$

Moreover, if $N_{q}(A) \geq q-1$ for any positive integer $q<2 l / n$, then one can take $d=1$. Otherwise, one can take $d$ to be the maximal number $q<2 l / n$ for which $N_{q}(A)<q-1$; in this case the set of all multiples of $d$ that fall into $I_{A}$ is contained already in $A_{d}^{*}$, where $A_{d}=\{a \in A: a \equiv 0(\bmod d)\}$.

In applications, one is often less concerned with the block (or progression) length and cares more about the density requirement and small values of $n$. For this reason we also provide a result which merely sharpens the constants of Theorem B.

TheOrem 2. Let $A \subseteq[1, l]$ be a set of $n=|A|$ integers satisfying

$$
n>10(l \ln n)^{1 / 2}
$$

(equivalently, $l<0.01 n^{2} / \ln n$ ). Then there are integers $y, z$, and $d \geq 1$ such that

$$
d<6 \frac{l}{n}, \quad z-y>\frac{n^{2}}{20}, \quad \frac{z-y}{y}>\frac{n^{2}}{115 l},
$$

and $\{y d,(y+1) d, \ldots, z d\} \subseteq A^{*}$.

Since the factor 10 (in the condition $n>10(l \ln n)^{1 / 2}$ ) is often more important than other constants, we give yet another version of this theorem.

Theorem $2^{\prime}$. Let $A \subseteq[1, l]$ be a set of $n=|A| \geq 3803$ integers satisfying

$$
n>7(l \ln n)^{1 / 2} \text {. }
$$

Then there are integers $y, z$, and $d \geq 1$ such that

$$
d<5 \frac{l}{n}, \quad z-y>\frac{n^{2}}{405}, \quad \frac{z-y}{y}>\frac{n^{2}}{2425 l},
$$

and $\{y d,(y+1) d, \ldots, z d\} \subseteq A^{*}$.

Remark. Observe that for any fixed $\varepsilon>0$ and $n$ large enough, the condition $n>7(l \ln n)^{1 / 2}$ follows from $n>(7 / \sqrt{2}+\varepsilon)(l \ln l)^{1 / 2}$; furthermore, $7 / \sqrt{2} \approx 4.949$.

3. The method. We prove Theorems 2 and $2^{\prime}$ in Section 5 and then derive Theorem 1 in Sections 6 and 7. Our proof of Theorems 2 and $2^{\prime}$ follows the lines of [S94] where their prototype, Theorem B, is established. 
To prove Theorem B, Sárközy uses a result about arithmetic progressions in the set

$$
h \cdot A:=\left\{a_{1}+\ldots+a_{h}: a_{1}<\ldots<a_{h}, a_{1}, \ldots, a_{h} \in A\right\}
$$

(all integers representable as a sum of precisely $h$ pairwise distinct elements of $A$ ).

Theorem C (Sárközy, [S94, Theorem 3]). Let $A \subseteq[1, l]$ be a set of $n=$ $|A|$ integers such that

$$
l>2500, \quad n>100(l \ln l)^{1 / 2} .
$$

Write $L=\ln (13 l / n)$. Then for every integer $M$ satisfying

$$
l \leq M \leq 10^{-4} \frac{n^{2}}{L}
$$

there exist positive integers $d$ and $h$ such that

$$
d<4828 \frac{l}{n}, \quad h<8496 \frac{M}{n}
$$

and the set $h \cdot A$ contains an $M$-term homogeneous arithmetic progression of difference $d$.

As shown in [S94], this theorem is best possible save for the constants and logarithmic factors.

To upgrade Theorem B to Theorems 2 and $2^{\prime}$ we establish in Section 4 a refined version of Theorem $\mathrm{C}$.

Theorem 3. Let $A \subseteq[1, l]$ be a set of $n=|A|$ integers. Write $L=$ $\ln (4 l / n)$. Then for every $M$ satisfying

$$
4 l \leq M \leq \frac{n^{2}}{12 L}
$$

there exist positive integers $d$ and $h$ such that

$$
d<4 \frac{l}{n}, \quad h<6 \frac{M}{n}
$$

and the set $h \cdot A$ contains a homogeneous arithmetic progression of difference $d$ and at least $M+1$ terms. Moreover, $d$ and $h$ can be so chosen that $2 d$ divides $h$.

Remarks. 1. Though in Theorem 3 we do not impose any explicit restrictions on $l$ or $n$, some are implied by the assumptions. For instance, from $l \geq n$ it follows that $L \geq \ln 4>4 / 3$, whence $n^{2} \geq 48 l L>64 n, n>64$; we use this observation in the proof in Section 4.

2. Some further minor improvements of the constants are possible. Also, there is the usual trade-off: some of the constants can be improved at the expense of the others. In fact, for any $\varepsilon>0$ we can find $c=c(\varepsilon)>0$ and $C=C(\varepsilon)>0$ such that if $c l \leq M \leq n^{2} /(C L)$, then $d$ and $h$ exist so that $d<(1+\varepsilon) l / n$ and $h<(1+\varepsilon) M / n$. 
Once Theorem 3 is proven, Theorems 2 and $2^{\prime}$ are relatively easy to deduce; this is done in Section 5.

Theorem 1 is derived from Theorem 2 in Sections 6 and 7. The proof uses some ideas from [AF88, C99]. Corollary 1 is proven in Section 8.

The reason why we believe that strengthening Theorems A and B is worthwhile is that they are used in the proofs of many important results in additive number theory. Replacing these theorems with Theorems 1, 2, and $2^{\prime}$ can lead to improvements in these results. We give an example in the Appendix.

Notation. Throughout the rest of the paper we continue to use the above introduced notation, unless indicated otherwise. Specifically, $A$ is a finite, non-empty set of positive integers; $l$ is a real number such that $A \subseteq[1, l]$; the cardinality of $A$ is $n=|A|$; the sum of the elements of $A$ is denoted by $\sigma(A)$; the number of elements of $A$ not divisible by an integer $q \geq 1$ is denoted by $N_{q}(A)$; the set of all numbers representable as a sum of precisely $h$ distinct elements of $A$ is $h \cdot A$, and $A^{*}=\bigcup_{h=0}^{n} h \cdot A$ is the subset sum set of $A$; finally, $\lambda=280 l / n^{2}$. Also, we write $h A$ for the set of all numbers representable as a sum of precisely $h$ not necessarily distinct elements of $A$ :

$$
h A=\left\{a_{1}+\ldots+a_{h}: a_{1}, \ldots, a_{h} \in A\right\} .
$$

4. Fixed number of summands: proof of Theorem 3. Our proof of Theorem 3 employs the same idea as that of Theorem C. However, we replace the key component of the proof, Sárközy's theorem [S89, Theorem 1], by the following result of ours.

Theorem 4 (Lev, [Le97, Theorem 3(ii)]). Let $S \subseteq[0, l]$ be a set of $|S|$ $\geq 3$ integers such that $0, l \in S$ and $\operatorname{gcd}(S)=1$. Write

$$
k=\left\lfloor\frac{l-1}{|S|-2}\right\rfloor, \quad \varrho=(k+1)(|S|-2)+2-l .
$$

Suppose that $h \geq 3 k$ is an integer number. Then $h S$ contains a block of $(h-k) l+k \varrho+1$ consecutive integers.

Another new ingredient we introduce in the proof is the following lemma.

Lemma 1. Let $A$ be a set of $n=|A|$ integers. For $t=1,2, \ldots$ denote by $S_{t}$ the set of all integers $s \in 2 A$ which have at least $t$ representations as $s=a_{1}+a_{2}\left(a_{1}, a_{2} \in A\right)$, and let $N_{t}=\left|S_{t}\right|$ be the cardinality of $S_{t}$. Then

$$
N_{1}+\ldots+N_{\tau} \geq 2 n \tau-\tau^{2}
$$

for any $\tau=1, \ldots, n$.

REMARK. The case $\tau=n$ is immediate (with equality sign) and was utilized in the original argument of Sárközy. The case $\tau=1$ is easy. 
Proof of Lemma 1. The shortest way to prove the lemma is to derive it from a result of Pollard [P74] which says that if $p$ is a prime number, $A_{1}$ and $A_{2}$ are non-empty sets of residues modulo $p$, and $N_{t}$ is the number of residues modulo $p$ with at least $t$ representations as $a_{1}+a_{2}\left(a_{i} \in A_{i}\right)$, then

$$
N_{1}+\ldots+N_{\tau} \geq \tau \min \left\{\left|A_{1}\right|+\left|A_{2}\right|-\tau, p\right\}
$$

for any $\tau=1, \ldots, \min \left\{\left|A_{1}\right|,\left|A_{2}\right|\right\}$. Now choose $A_{1}=A_{2}$ to be the canonical image of $A$ in $\mathbb{Z} / p \mathbb{Z}$, where $p$ is a sufficiently large prime number.

From now on, we adopt $S_{t}$ and $N_{t}$ (not to be confused with $N_{q}(A)$ !) as a standard notation.

Corollary 2. Let $A, n, l$, and $L$ be as in Theorem 3. Then there exists $t \in[1, n]$ such that

$$
N_{t}>\frac{n}{2} \max \left\{\frac{n}{t L}, 1\right\}+2 .
$$

Proof. Assume the opposite:

$$
N_{t} \leq \frac{n}{2} \max \left\{\frac{n}{t L}, 1\right\}+2, \quad t=1, \ldots, n .
$$

Set $\tau=\lfloor 3 n / 4\rfloor$. Taking into account that $N_{t}<2 l$ for any $t$, by Lemma 1 and (2) we get

$$
\begin{aligned}
2 n \tau-\tau^{2} & \leq N_{1}+\ldots+N_{\tau} \\
& <\sum_{1 \leq t \leq n^{2} /(4 l L)} 2 l+\sum_{n^{2} /(4 l L)<t \leq n / L}\left(\frac{1}{2} \cdot \frac{n^{2}}{t L}+2\right)+\sum_{n / L<t \leq \tau}\left(\frac{1}{2} n+2\right) \\
& \leq \frac{n^{2}}{2 L}+2(\tau-1)+\frac{1}{2} \cdot \frac{n^{2}}{L}\left(\frac{4 l L}{n^{2}}+\ln \frac{4 l}{n}\right)+\frac{1}{2} n\left(\tau+1-\frac{n}{L}\right) \\
& =\frac{1}{2} n^{2}+\frac{1}{2} n \tau+\left(2 l+\frac{1}{2} n+2 \tau-2\right) .
\end{aligned}
$$

(We use here the estimate $\sum_{P<t \leq Q} t^{-1} \leq P^{-1}+\ln (Q / P)$. Also, the number of summands in $\sum_{n / L<t \leq \tau}$ is at most $\tau+1-n / L$, as $\tau+1>3 n / 4>n / L$ in view of $L>4 / 3$.) This implies that

$$
\begin{aligned}
\frac{1}{16} n^{2} & <\left(\tau-\frac{3}{4} n\right)^{2}+\left(2 l+\frac{1}{2} n+2 \tau-2\right)<2 l+\frac{1}{2} n+2 \tau-1 \\
& \leq 2 l+2 n-1, \\
l & >\frac{n^{2}}{32}-n>\frac{n^{2}}{64}>\frac{n^{2}}{48 L},
\end{aligned}
$$

contrary to the assumption that $4 l \leq M \leq n^{2} /(12 L)$.

Our next lemma is parallel to [S94, Lemma 1]. 
Lemma 2. Let $A$ be a set of integers, and suppose that $h<\lceil(t+3) / 4\rceil$ is a positive integer. Then $h S_{t} \subseteq 2 h \cdot A$.

Sketch of the proof. Use induction on $j=1, \ldots, h$ to show that $j S_{t} \subseteq$ $2 j \cdot A$. Let $s_{1}+\ldots+s_{j-1}+s_{j}$ be an element of $j S_{t}$. By the induction hypothesis, there is a representation $s_{1}+\ldots+s_{j-1}=a_{1}+\ldots+a_{2 j-2}$ with $a_{i} \in A$ all distinct, and the assumption on $h$ ensures that $s_{j}$ has a representation $s_{j}=a^{\prime}+a^{\prime \prime}$ with $a^{\prime} \neq a^{\prime \prime}$ and both $a^{\prime}$ and $a^{\prime \prime}$ distinct from all $a_{i}$.

Proof of Theorem 3. We choose $t \in[1, n]$ satisfying (1), and we put

$$
d:=\operatorname{gcd}\left(S_{t}-S_{t}\right), \quad S_{t}^{\prime}:=\left\{d^{-1}\left(s-\min \left(S_{t}\right)\right): s \in S_{t}\right\}, \quad l^{\prime}:=\max \left(S_{t}^{\prime}\right) .
$$

Thus, $S_{t}^{\prime} \subseteq\left[0, l^{\prime}\right], 0, l^{\prime} \in S_{t}^{\prime}, \operatorname{gcd}\left(S_{t}^{\prime}\right)=1$, and $\left|S_{t}^{\prime}\right|=N_{t}$. Set

$$
k:=\left\lfloor\frac{l^{\prime}-1}{N_{t}-2}\right\rfloor, \quad \varrho:=(k+1)\left(N_{t}-2\right)+2-l^{\prime} .
$$

For future reference we note that

$$
k\left(N_{t}-2\right)<l^{\prime} \leq \frac{1}{d}\left(\max \left(S_{t}\right)-\min \left(S_{t}\right)\right)<\frac{2 l}{d},
$$

hence by (1) and $4 l \leq M \leq n^{2} /(12 L)$,

$$
\begin{aligned}
& d<\frac{2 l}{k\left(N_{t}-2\right)}<\frac{4 l}{k n} \min \left\{\frac{t L}{n}, 1\right\} \\
& k<\frac{4 l}{d n} \min \left\{\frac{t L}{n}, 1\right\} \\
& t>\frac{k d n^{2}}{4 l L} \geq \frac{3 k d M}{l} \geq 12 k d .
\end{aligned}
$$

We now define

$$
h_{0}:=\max \left\{2 k,\left\lceil\frac{M-k \varrho}{l^{\prime}}\right\rceil\right\}+k+\delta, \quad h=2 h_{0},
$$

where $\delta \in[0, d-1]$ is chosen so that $d \mid h_{0}$. As $h_{0} \geq 3 k$, by Theorem 4 the set $h_{0} S_{t}^{\prime}$ contains a block of at least $\left(h_{0}-k\right) l^{\prime}+k \varrho+1 \geq\left\lceil(M-k \varrho) / l^{\prime}\right\rceil l^{\prime}+k \varrho+1 \geq$ $M+1$ consecutive integers, hence $h_{0} S_{t}$ contains a homogeneous arithmetic progression of difference $d$ and at least $M+1$ terms. To complete the proof it suffices to show that

$$
h_{0}<\min \left\{\frac{t}{4}, 3 \frac{M}{n}\right\}
$$

(the estimate $d<4 l / n$ was already established in (4)): then by Lemma 2 we have $h \cdot A=2 h_{0} \cdot A \supseteq h_{0} S_{t}$, whence $h \cdot A$ contains a homogeneous arithmetic progression of difference $d$ and at least $M+1$ terms. 
Evidently, (7) will follow from

$$
3 k+d-1<\min \left\{\frac{t}{4}, 3 \frac{M}{n}\right\},
$$

and

$$
\frac{M-k \varrho}{l^{\prime}}+k+d<\min \left\{\frac{t}{4}, 3 \frac{M}{n}\right\} .
$$

To prove (8) we observe that by (4),

$$
3 k+d-1<3 k+\frac{4 l}{k n} \min \left\{\frac{t L}{n}, 1\right\}-1 .
$$

We consider the right-hand side as a function of $k \in[1, K]$, where

$$
K:=\frac{4 l}{n} \min \left\{\frac{t L}{n}, 1\right\}
$$

(cf. (5)) and we denote this function by $f(k)$. Now (8) follows from the fact that $f$ is convex,

$$
f(1)=\frac{4 l}{n} \min \left\{\frac{t L}{n}, 1\right\}+2 \leq \min \left\{\frac{t}{12}+2, \frac{M}{n}+2\right\} \leq \min \left\{\frac{t}{4}, 3 \frac{M}{n}\right\}
$$

(notice that $t>12$ by (6)), and

$$
f(K)=\frac{12 l}{n} \min \left\{\frac{t L}{n}, 1\right\} \leq \min \left\{\frac{t}{4}, 3 \frac{M}{n}\right\} .
$$

Finally, to prove (9) we consider two cases: $k=1$ and $k \geq 2$.

If $k=1$ then by (3) and (1),

$$
\begin{aligned}
\frac{M-k \varrho}{l^{\prime}}+k+d & <\frac{M-\varrho+2 l}{l^{\prime}}+1=\frac{M-\left(2 N_{t}-2-l^{\prime}\right)+2 l}{l^{\prime}}+1 \\
& \leq \frac{3 M / 2-2\left(N_{t}-1\right)}{l^{\prime}}+2<\frac{3 M / 2}{N_{t}-2} \\
& <3 \frac{M}{n} \min \left\{\frac{t L}{n}, 1\right\} \leq \min \left\{\frac{t}{4}, 3 \frac{M}{n}\right\} .
\end{aligned}
$$

If $k \geq 2$ then by (3) and (5),

$$
\begin{aligned}
\frac{M-k \varrho}{l^{\prime}}+k+d & <\frac{M+2 l}{l^{\prime}}+k<\frac{M+2 l}{n} \min \left\{\frac{t L}{n}, 1\right\}+k \\
& <\frac{M+6 l}{n} \min \left\{\frac{t L}{n}, 1\right\}<3 \frac{M}{n} \min \left\{\frac{t L}{n}, 1\right\} \\
& \leq \min \left\{\frac{t}{4}, 3 \frac{M}{n}\right\} .
\end{aligned}
$$


5. Proof of Theorems 2 and $\mathbf{2}^{\prime}$. We only sketch the proof of Theorem 2, which essentially follows that of Theorem B, and indicate how it is to be modified to obtain Theorem $2^{\prime}$.

We need a lemma which is a minor variation of [S94, Lemma 3].

Lemma 3. Let $U, V$, and $d$ be positive integers and let $B \subseteq[U, V]$ be a set of integers. Then for any $s \in B^{*}$ such that $d \mid s, s \leq U(|B|-d)$ there exists $s^{\prime} \in B^{*}$ satisfying

$$
d \mid s^{\prime}, \quad 0<s^{\prime}-s \leq V d .
$$

Proof. Choose $S \subseteq B$ such that $s=\sum_{b \in S} b$. As

$$
U|S| \leq s \leq U(|B|-d)
$$

we have $|B \backslash S| \geq d$, and therefore there is a set $S_{0} \subseteq B \backslash S$ of cardinality $0<\left|S_{0}\right| \leq d$ such that $\sum_{b \in S_{0}} b \equiv 0(\bmod d)$. We define $S^{\prime}:=S \cup S_{0}$ and $s^{\prime}:=\sum_{b \in S^{\prime}} b$; then

$$
s^{\prime}-s=\sum_{b \in S_{0}} b \leq V\left|S_{0}\right| \leq V d
$$

and the validity of $d \mid s^{\prime}$ is obvious from the construction.

To prove Theorem 2 we write $A=\left\{a_{1}, \ldots, a_{n}\right\}$ (the elements being numbered in increasing order) and set

$$
j:=\lfloor 0.7 n\rfloor, \quad A_{1}:=\left\{a_{1}, \ldots, a_{j}\right\}, \quad A_{2}:=\left\{a_{j+1}, \ldots, a_{n}\right\} .
$$

(For Theorem $2^{\prime}$, set $j:=\lfloor 0.99 n\rfloor$.) We apply Theorem 3 to the set $A_{1} \subseteq$ $\left[1, a_{j}\right]$ of cardinality $j=\left|A_{1}\right|$ and with $M=4 l$. (Verification of the condition $M \leq\left|A_{1}\right|^{2} /(12 L)$, or equivalently $j^{2} \geq 48 l \ln \left(4 a_{j} / j\right)$, is left to the reader; hint: $4 a_{j} / j<4 l / j \leq n$, and $j^{2}>0.49 n^{2}-1.4 n$.) We conclude that there exist integers $d, h \geq 1$ and $y$ such that

$$
\{y d,(y+1) d, \ldots,(y+4 l) d\} \subseteq h \cdot A_{1}
$$

and

$$
d<4 \frac{a_{j}}{j}, \quad h<24 \frac{l}{j}
$$

we observe, moreover, that (11) implies

$$
d<4 \frac{l}{j}<6 \frac{l}{n}
$$

and

$$
y d \leq h a_{j}<24 \frac{l a_{j}}{j} .
$$

Next, applying Lemma 3 to the set $B=A_{2} \subseteq\left[a_{j}, l\right]$ we see that for any $s \in A_{2}^{*}$ such that $d \mid s$ and $s \leq a_{j}(n-j-d)$ there exists $s^{\prime} \in A_{2}^{*}$ such that 
$d \mid s^{\prime}$ and $s^{\prime}-s \leq l d$. Along with (10) this shows that

$$
\left\{y d,(y+1) d, \ldots,\left(\left\lfloor a_{j}(n-j-d) / d\right\rfloor+1\right) d+(y+4 l) d\right\} \subseteq A_{1}^{*}+A_{2}^{*}=A^{*} .
$$

We put

$$
z:=\left\lfloor\frac{a_{j}(n-j-d)}{d}\right\rfloor+1+(y+4 l)
$$

so that by (11),

$$
\begin{aligned}
z-y & >\frac{a_{j}(n-j)}{d}+\left(4 l-a_{j}\right)>\frac{a_{j}(n-j)}{d} \\
& >\frac{1}{4} j(n-j) \geq \frac{0.21}{4} n^{2}>\frac{1}{20} n^{2} .
\end{aligned}
$$

It remains to notice that by (12),

$$
\frac{z-y}{y}>\frac{a_{j}(n-j)}{y d}>\frac{j(n-j)}{24 l} \geq \frac{0.21}{24} \cdot \frac{n^{2}}{l}>\frac{n^{2}}{115 l} .
$$

REMARK. It is clear that the above argument actually gives slightly better constants than those indicated in the formulation of Theorems 2 and $2^{\prime}$. In particular, the reader can easily verify that our proof of Theorem 2 remains valid if the assumption $n \geq 10(l \ln n)^{1 / 2}$ is relaxed to $n \geq$ $9.95(l \ln n)^{1 / 2}$. This observation will be used in Section 7 to avoid further loss of accuracy.

6. Auxiliary results on addition in $\mathbb{Z}_{q}$. We say (modifying slightly the terminology of [C90, C99]) that a set of integers $A$ is q-complete if $A^{*}$ $(\bmod q)=\mathbb{Z}_{q}$; in other words, if all residues modulo $q$ are represented in $A^{*}$. In the spirit of the two papers just mentioned, we establish here a sufficient condition for $q$-completeness.

Lemma 4. Let $A$ be a finite set of integers and let $q \geq 1$ be an integer. Suppose that $N_{d}(A) \geq d-1$ for any $d \mid q$. Then $A$ is q-complete.

Proof. We can assume that $A$ is finite, and we use induction by $n=|A|$. Write $A=\left\{a_{1}, \ldots, a_{n}\right\}$ and $A_{i}=\left\{a_{1}, \ldots, a_{i}\right\}(1 \leq i \leq n)$ and let overlined characters denote canonical images in $\mathbb{Z}_{q}$. Suppose that $a_{i}$ are so numbered that $\bar{A}_{1}^{*} \varsubsetneqq \ldots \varsubsetneqq \bar{A}_{j}^{*}$ and that either $A_{j}=A$, or $\left(\bar{A}_{j} \cup\{\bar{a}\}\right)^{*}=\bar{A}_{j}^{*}$ for any $a \in A \backslash A_{j}$. In the former case we have $j=n$ and accordingly

$$
\left|\bar{A}^{*}\right|=\left|\bar{A}_{j}^{*}\right| \geq j+1=|A|+1 \geq N_{q}(A)+1 \geq q,
$$

which proves the assertion. We assume, therefore, that the second possibility holds, which translates easily into $\bar{A}_{j}^{*}+\bar{a}=\bar{A}_{j}^{*}$ (for any $a \in A \backslash A_{j}$ ).

Consider the subgroup of all $g \in \mathbb{Z}_{q}$ satisfying $\bar{A}_{j}^{*}+g=\bar{A}_{j}^{*}$ and write this subgroup as $d \mathbb{Z}_{q}$, where $d$ is a divisor of $q$. (This subgroup is often called the period or stabilizer of $\bar{A}_{j}^{*}$.) The condition $\bar{A}_{j}^{*}+\bar{a}=\bar{A}_{j}^{*}$ means that $\bar{a} \in d \mathbb{Z}_{q}$, that is, $d \mid a$, and it follows that $\delta \mid a$ for any divisor $\delta \mid d$. This shows that 
$N_{\delta}\left(A_{j}\right)=N_{\delta}(A) \geq \delta-1$, provided $\delta \mid d$. By the induction hypothesis, $A_{j}$ is $d$-complete; that is, $\bar{A}_{j}^{*}$ contains representatives of all $d \mathbb{Z}_{q}$-cosets. Now $\bar{A}_{j}^{*}=\bar{A}_{j}^{*}+d \mathbb{Z}_{q}=\mathbb{Z}_{q}$, implying the result.

Corollary 3. Suppose that $A \subseteq \mathbb{Z}$ satisfies $N_{q}(A) \geq q-1$ for any $q \leq Q$. Then $A$ is $q$-complete for any $q \leq Q$.

Lemma 5 (Chaimovich, [C99, Lemma 2.3]). If $A$ is q-complete then there is a subset $A_{0} \subseteq A$ of cardinality $\left|A_{0}\right| \leq q-1$ which is also q-complete.

Proof. As in the proof of the previous lemma, we use overlined characters to denote canonical images in $\mathbb{Z}_{q}$. Let $A_{0}=\left\{a_{1}, \ldots, a_{k}\right\} \subseteq A$ be a subset of minimum cardinality such that $\bar{A}_{0}^{*}=\mathbb{Z}_{q}$, and let $A_{j}=\left\{a_{1}, \ldots, a_{j}\right\}(j=$ $1, \ldots, k)$. If $\bar{A}_{1}^{*} \varsubsetneqq \ldots \varsubsetneqq \bar{A}_{k}^{*}$ then $q=\left|\bar{A}_{k}^{*}\right| \geq k+1$, as required. Otherwise, there is an index $j \leq k-1$ such that $\bar{A}_{j}^{*}=\bar{A}_{j+1}^{*}$. But in this case we would have

$$
\begin{aligned}
\bar{A}_{0}^{*} & =\bar{A}_{j+1}^{*}+\left\{\bar{a}_{j+2}, \ldots, \bar{a}_{k}\right\}^{*}=\bar{A}_{j}^{*}+\left\{\bar{a}_{j+2}, \ldots, \bar{a}_{k}\right\}^{*} \\
& =\left\{\bar{a}_{1}, \ldots, \bar{a}_{j}, \bar{a}_{j+2}, \ldots, \bar{a}_{k}\right\}^{*}
\end{aligned}
$$

contradicting minimality of $A_{0}$.

\section{Proof of Theorem 1}

Lemma 6. For any $C, \varepsilon>0$ and $K \geq 2$ there exists $n_{0}=n_{0}(C, \varepsilon, K)$ with the following property. Let $Q \geq 2$ and let $A \subseteq[1, l]$ be a set of integers of cardinality $n=|A| \geq C(l \ln l)^{1 / 2}, n \geq n_{0}$, satisfying $N_{q}(A) \geq K q$ for all $q \in[2, Q]$. Then there is a subset $A_{0} \subseteq A$ such that

(i) $K^{-1} n<\left|A_{0}\right|<(1+\varepsilon) K^{-1} n$;

(ii) $\sigma\left(A_{0}\right)<(1+\varepsilon) K^{-1} \sigma(A)$;

(iii) $N_{q}\left(A_{0}\right) \geq q$ for all $q \in[2, Q]$.

Proof. Fix arbitrarily $p \in\left(K^{-1},(1+\varepsilon) K^{-1}\right), p<1$ and set

$$
\delta:=\frac{1}{2} \min \left\{1-(p K)^{-1},(1+\varepsilon)(p K)^{-1}-1\right\} .
$$

Define $P(q):=e^{-\delta^{2} p K q / 2}$. As the series $\sum_{q=2}^{\infty} P(q)$ converges, there is an integer $Q_{0}=Q_{0}(p, K)$ such that $\sum_{q=Q_{0}+1}^{\infty} P(q)<0.1$.

The set $A_{0}$ will be comprised of two parts. First, for each integer $q \in$ $\left[2, Q_{0}\right]$ we choose $q$ arbitrary elements of $A$, not divisible by $q$, and we denote by $A_{1}$ the set of all elements chosen. Plainly, $\left|A_{1}\right|<\frac{1}{2} Q_{0}\left(Q_{0}+1\right)$. Second, we let $A_{2}$ be the random subset of $A$ to which any $a \in A$ belongs with probability $p$ (with all $a \in A$ being selected independently). Thus $\left|A_{2}\right|$ is distributed binomially with parameters $n$ and $p$, and $\mathrm{E}\left|A_{2}\right|=p n$. Eventually, we define $A_{0}:=A_{1} \cup A_{2}$. 
Using an estimate for the right tail of binomial distribution (see, for instance, [JŁR, Theorems 2.1 and 2.10]) we get

$$
\operatorname{Pr}\left\{\left|A_{2}\right| \leq(1-\delta) p n\right\}=\operatorname{Pr}\left\{\left|A_{2}\right| \leq(1-\delta) \mathrm{E}\left|A_{2}\right|\right\} \leq e^{-\delta^{2} \mathrm{E}\left|A_{2}\right| / 2}=e^{-\delta^{2} p n / 2} .
$$

As $(1-\delta) p>K^{-1}$, for $n$ large enough this implies

$$
\operatorname{Pr}\left\{\left|A_{0}\right| \leq K^{-1} n\right\} \leq \operatorname{Pr}\left\{\left|A_{2}\right| \leq(1-\delta) p n\right\}<0.1 .
$$

Similarly, in view of $\left|A_{1}\right|=O_{p, K}(1)$ we have

$$
\operatorname{Pr}\left\{\left|A_{0}\right| \geq(1+\varepsilon) K^{-1} n\right\} \leq \operatorname{Pr}\left\{\left|A_{2}\right| \geq(1+\delta) p n\right\}<0.1 ;
$$

therefore, (i) fails with probability at most 0.2 .

Furthermore, $\sigma\left(A_{2}\right):=\sum_{a \in A_{2}} a$ is a random variable with expectation $\mathrm{E} \sigma\left(A_{2}\right)=p \sigma(A)$ and variance

$$
\operatorname{Var} \sigma\left(A_{2}\right)=p(1-p) \sum_{a \in A} a^{2}<p l \sigma(A) .
$$

By Chebyshev's inequality we have

$$
\begin{aligned}
\operatorname{Pr}\left\{\sigma\left(A_{2}\right) \geq(1+\delta) p \sigma(A)\right\}=\operatorname{Pr}\left\{\sigma\left(A_{2}\right) \geq(1+\delta) \mathrm{E} \sigma\left(A_{2}\right)\right\} \\
<\frac{p l \sigma(A)}{\left(\delta \mathrm{E} \sigma\left(A_{2}\right)\right)^{2}}=\left(\delta^{2} p\right)^{-1} \frac{l}{\sigma(A)}<2\left(\delta^{2} p\right)^{-1} \frac{l}{n^{2}}<0.1,
\end{aligned}
$$

provided that $n$ is large enough. As $(1+\delta) p<(1+\varepsilon) K^{-1}$ and in view of $\sigma\left(A_{1}\right)=O(l)=o(\sigma(A))$ this implies that

$$
\operatorname{Pr}\left\{\sigma\left(A_{0}\right) \geq(1+\varepsilon) K^{-1} \sigma(A)\right\}<0.1 ;
$$

that is, (ii) fails with probability at most 0.1 .

Finally, we take care of (iii). For any integer $q \in\left[Q_{0}+1, Q\right]$ the random variable $N_{q}\left(A_{2}\right)$ is distributed binomially with parameters $N_{q}(A)$ and $p$, whence in view of $q \leq K^{-1} N_{q}(A)=(K p)^{-1} \mathrm{E} N_{q}\left(A_{2}\right)<(1-\delta) \mathrm{E} N_{q}\left(A_{2}\right)$ we get

$$
\begin{aligned}
\operatorname{Pr}\left\{N_{q}\left(A_{2}\right)<q\right\} & \leq \operatorname{Pr}\left\{N_{q}\left(A_{2}\right)<(1-\delta) \mathrm{E} N_{q}\left(A_{2}\right)\right\} \\
& <e^{-\delta^{2} \mathrm{E} N_{q}\left(A_{2}\right) / 2} \leq e^{-\delta^{2} p K q / 2}=P(q)
\end{aligned}
$$

(estimating the left binomial tail). It follows that the probability that there is a value of $q \in\left[Q_{0}+1, Q\right]$ such that $N_{q}\left(A_{2}\right)<q$, and therefore the probability that there is a value of $q \in[2, Q]$ such that $N_{q}\left(A_{0}\right)<q$, is at most $\sum_{q=Q_{0}+1}^{\infty} P(q)<0.1$ (by the choice of $Q_{0}$ ). Therefore, (iii) fails with probability 0.1 at most.

We see that the probability that any of conditions (i)-(iii) fails to hold is $0.2+0.1+0.1<1$ at most. Hence, with a positive probability $A_{0}$ satisfies all the properties required. 
Proposition 1. There exist positive constants $K$ and $n_{0}$ with the following property. Let $A \subseteq[1, l]$ be a set of $n=|A| \geq n_{0}$ integers such that

$$
n \geq 9.96(l \ln n)^{1 / 2}
$$

and $N_{q}(A) \geq K q$ for any integer $q \in[2,7 l / n]$. Then there are integers $y$ and $z$ such that $[y, z] \subseteq A^{*}$ and

$$
z-y>\frac{n^{2}}{21}, \quad \frac{z-y}{y}>\frac{n^{2}}{138 l} .
$$

Proof. Choose positive $K$ and $\varepsilon$ so that the former is sufficiently large and the latter is sufficiently small. By Lemma 6 and Corollary 3 there is a subset $A_{0} \subseteq A$ of cardinality $\left|A_{0}\right|<(1+\varepsilon) K^{-1} n<0.001 n$ which is $q$ complete for any $q \leq 7 l / n$. Write $A_{1}:=A \backslash A_{0}$, so that $n_{1}:=\left|A_{1}\right|>0.999 n$. We have

$$
n_{1}>0.999 \cdot 9.96(l \ln n)^{1 / 2}>9.95\left(l \ln n_{1}\right)^{1 / 2}
$$

and by Theorem 2 as applied to the set $A_{1}$ (see also the Remark at the end of Section 5), there exist integers $y_{1}, z_{1}$, and $d \geq 1$ such that $\left\{y_{1} d\right.$, $\left.\left(y_{1}+1\right) d, \ldots, z_{1} d\right\} \subseteq A_{1}^{*}$ and

$$
d<6 \frac{l}{n_{1}}<7 \frac{l}{n}, \quad z_{1}-y_{1}>\frac{n_{1}^{2}}{20}>\frac{n^{2}}{21}, \quad \frac{z_{1}-y_{1}}{y_{1}}>\frac{n_{1}^{2}}{115 l}>\frac{n^{2}}{116 l} .
$$

If $d=1$ the assertion follows. Assuming that $d \geq 2$, find $B_{0} \subseteq A_{0}$ which is $d$-complete and such that $\left|B_{0}\right| \leq d-1$ : this is possible by Lemma 5 . Notice that to any integer $r \in[0, d-1]$ there corresponds $k_{r} \in[0, l-1]$ such that $r+k_{r} d \in B_{0}^{*}$. Now set $y:=\left(y_{1}+l\right) d$ and $z:=z_{1} d$; then

$$
z-y=\left(z_{1}-y_{1}-l\right) d>z_{1}-y_{1}>n^{2} / 21,
$$

as $z_{1}-y_{1}>n^{2} / 21>2 l \geq l d /(d-1)$. Furthermore, let $\kappa=116 / 21$. If $y_{1} \geq \kappa l$ then

$$
\frac{z-y}{y}=\frac{z_{1}}{y_{1}+l}-1 \geq \frac{\kappa}{\kappa+1} \cdot \frac{z_{1}}{y_{1}}-1>\frac{\kappa}{\kappa+1} \cdot \frac{n^{2}}{116 l}-1>\frac{n^{2}}{138 l},
$$

and if $y_{1} \leq \kappa l$ then

$$
\frac{z-y}{y}=\frac{z_{1}}{y_{1}+l}-1 \geq \frac{1}{\kappa+1} \cdot \frac{z_{1}}{l}-1>\frac{1}{\kappa+1} \cdot \frac{n^{2}}{21 l}-1>\frac{n^{2}}{138 l} .
$$

To complete the proof we show that $[y, z] \subseteq B_{0}^{*}+A_{1}^{*}$. Indeed, given $x \in[y, z]$ write $x=r+k d$ where $r \in[0, d-1]$ and $y_{1}+l \leq k \leq z_{1}$. Then $x=$ $\left(r+k_{r} d\right)+\left(k-k_{r}\right) d$ and it suffices to notice that $y_{1} \leq k-l<k-k_{r} \leq z_{1}$.

As a next step, we show that the condition $N_{q}(A) \geq K q$ of Proposition 1 can be relaxed considerably. 
Proposition 2. Let $A \subseteq[1, l]$ be a set of $n=|A| \geq n_{0}$ integers, where $n_{0}$ is a sufficiently large absolute constant. Suppose that

$$
n \geq 9.96(l \ln n)^{1 / 2}
$$

and that $N_{q}(A) \geq q-1$ for any integer $q<2 l / n$. Then there are integers $y$ and $z$ such that $[y, z] \subseteq A^{*}$ and

$$
z-y>\frac{n^{2}}{21}, \quad \frac{z-y}{y}>\frac{n^{2}}{138 l} .
$$

Proof. Let $K$ be the constant of Proposition 1. If $N_{q}(A) \geq K q$ for all integer $q \in[2,7 l / n]$ then the result follows from Proposition 1. Otherwise, denote by $q$ the maximal number such that $2 \leq q \leq 7 l / n$ and $N_{q}(A)<K q$. Let $A_{0}$ be the set of all elements of $A$, not divisible by $q$, and let $A_{1}$ be the set of integers such that $A \backslash A_{0}=\left\{q a: a \in A_{1}\right\}$. Evidently, we have $A_{1} \subseteq\left[1, l_{1}\right]$ where $l_{1}=\lfloor l / q\rfloor \leq l / 2$. Furthermore, the cardinality of $A_{1}$ satisfies $n_{1}:=\left|A_{1}\right|>n-K q$, and if $n$ is large enough then $q<0.1 K^{-1} n$ and $n_{1}>0.9 n$.

We want to apply Proposition 1 to the set $A_{1}$. We have

$$
n_{1}>0.9 n \geq 0.9 \cdot 0.96(l \ln n)^{1 / 2}>0.96\left((l / 2) \ln n_{1}\right)^{1 / 2} \geq 0.96\left(l_{1} \ln n_{1}\right)^{1 / 2}
$$

and for Proposition 1 to be applicable it is necessary and sufficient that $N_{q_{1}}\left(A_{1}\right) \geq K q_{1}$ for any $q_{1} \in\left[2,7 l_{1} / n_{1}\right]$. Assume that this does not hold for some $q_{1}$. Then $q_{1} \leq 7 l /(0.9 n) \leq 0.1 K^{-1} n$ (again, assuming that $n$ is sufficiently large), hence $K\left(q+q_{1}\right)<0.2 n$ and therefore

$$
\frac{l}{q} \geq l_{1}>\left(n_{1}-K q_{1}\right) q_{1}>\left(n-K\left(q+q_{1}\right)\right) q_{1}>0.8 n q_{1}, \quad q q_{1}<2 \frac{l}{n} .
$$

Along with $N_{q q_{1}}(A)<K q+K q_{1} \leq K q q_{1}$ this contradicts maximality of $q$.

We see that the conditions of Proposition 1 are fulfilled and there exist integers $y_{1}$ and $z_{1}$ such that

$$
z_{1}-y_{1}>\frac{n_{1}^{2}}{21}>\frac{n^{2}}{26}, \quad \frac{z_{1}-y_{1}}{y_{1}}>\frac{n_{1}^{2}}{138 l_{1}}>\frac{n^{2}}{86 l}
$$

and $\left[y_{1}, z_{1}\right] \subseteq A_{1}^{*}$. Notice that the latter inclusion yields $\left\{y_{1} q,\left(y_{1}+1\right) q, \ldots, z_{1} q\right\}$ $\subseteq\left(A \backslash A_{0}\right)^{*}$.

We have assumed that $q \leq 7 l / n$ and we now observe that in fact, $q<2 l / n$ holds, for $\lfloor l / q\rfloor=l_{1} \geq n_{1}>0.9 n$. It follows that $A$, and therefore $A_{0}$ also, is $q$-complete and, as in the proof of Proposition 1, we conclude that $[y, z] \subseteq A^{*}$ with $y=\left(y_{1}+l\right) q$ and $z=z_{1} q$. Finally, we have

$$
z-y \geq 2\left(z_{1}-y_{1}-l\right) \geq 2\left(n^{2} / 26-l\right)>n^{2} / 21
$$

and to estimate $(z-y) / y=z_{1} /\left(y_{1}+l\right)-1$ one can define $\kappa=86 / 26$ and act as in the proof of Proposition 1. 
Proposition 3. Let $A \subseteq[1, l]$ be a set of $n=|A| \geq n_{0}$ integers, where $n_{0}$ is a sufficiently large absolute constant. Suppose that

$$
n \geq 20(l \ln n)^{1 / 2}
$$

and that $N_{q}(A) \geq 3 q$ for any integer $q \in[2,5 l / n]$. Then

$$
[\lambda \sigma(A),(1-\lambda) \sigma(A)] \subseteq A^{*} .
$$

Proof. Set $K=2.008$, fix $\varepsilon$ positive and small enough and find a subset $A_{0} \subseteq A$ satisfying conditions of Lemma 6 . Write $A_{1}:=A \backslash A_{0}$. We have

$$
n_{0}:=\left|A_{0}\right|>K^{-1} n \geq 9.96(l \ln n)^{1 / 2} \geq 9.96\left(l \ln n_{0}\right)^{1 / 2}
$$

and $N_{q}\left(A_{0}\right) \geq q-1$ for any $q \leq 5 l / n$, hence for any $q<2 l / n_{0}$. Proposition 2 shows, therefore, that there is an interval $[y, z] \subseteq A_{0}^{*}$ such that $z-y>$ $n_{0}^{2} / 21>n^{2} /\left(21 K^{2}\right)>l$ and $(z-y) / y>n_{0}^{2} /(138 l)$. Since the gaps between consecutive elements of $A_{1}^{*}$ do not exceed $l$, it follows that $\left[y, \sigma\left(A_{1}\right)+z\right] \subseteq$ $A_{0}^{*}+A_{1}^{*}=A^{*}$. We now observe that

$$
y<138 \frac{l}{n_{0}^{2}} z<138 K^{2} \frac{l}{n^{2}} \sigma\left(A_{0}\right)<138(1+\varepsilon) K \frac{l}{n^{2}} \sigma(A)<\lambda \sigma(A)
$$

and that

$$
\sigma\left(A_{1}\right)+z \geq \sigma(A)-\sigma\left(A_{0}\right)>\left(1-(1+\varepsilon) K^{-1}\right) \sigma(A)>\sigma(A) / 2 .
$$

Hence, $[\lambda \sigma(A), \sigma(A) / 2] \subseteq A^{*}$ and it remains to notice that $A^{*}$ is symmetric about $\sigma(A) / 2$ : if $s \in A^{*}$ then also $\sigma(A)-s \in A^{*}$ for any integer $s$.

Finally, we are ready to prove Theorem 1 . For this, we "improve" Proposition 3 in the same spirit as we did with Proposition 1.

Proof of Theorem 1. The beginning of the proof runs as that of Proposition 2. If for any $q \in[2,5 l / n]$ we have $N_{q}(A) \geq 3 q$, then the result follows at once by Proposition 3. Otherwise, let $q \in[2,5 l / n]$ be the maximal integer satisfying $N_{q}(A)<3 q$. Define $A_{0}$ to be the set of all elements of $A$ not divisible by $q$ (thus $\left|A_{0}\right|=N_{q}(A)<3 q$ ), and let $A_{1}$ be the set of integers such that $A \backslash A_{0}=\left\{q a: a \in A_{1}\right\}$. Evidently, we have $A_{1} \subseteq\left[1, l_{1}\right]$, where $l_{1}=\lfloor l / q\rfloor$, and $n_{1}:=\left|A_{1}\right|>n-3 q \geq 0.9 n$ for $n$ large enough, as then $q \leq 5 l / n \leq 0.03 n$. Therefore,

$$
n_{1}>0.9 n \geq 18(l \ln n)^{1 / 2}>20\left((l / 2) \ln n_{1}\right)^{1 / 2} \geq 20\left(l_{1} \ln n_{1}\right)^{1 / 2}
$$

and also $N_{q_{1}}\left(A_{1}\right) \geq 3 q_{1}$ for $q_{1} \in\left[2,5 l_{1} / n_{1}\right]$, as in the proof of Proposition 2 . By Proposition 3 we have $[y, z] \in A_{1}^{*}$ with $y$ and $z$ integer and satisfying $y \leq\left\lceil\lambda_{1} \sigma\left(A_{1}\right)\right\rceil, z \geq\left\lfloor\left(1-\lambda_{1}\right) \sigma\left(A_{1}\right)\right\rfloor$, where $\lambda_{1}=280 l_{1} / n_{1}^{2}$. Furthermore, $q \leq l / l_{1} \leq l / n_{1}<2 l / n$, whence $A_{0}$ is $q$-complete. It follows that $[(y+l) q, z q]$ $\in A^{*}$. Next, we have

$$
l q=2 \frac{l}{n_{1}^{2}} \cdot \frac{n_{1}^{2}}{2} q<3 \frac{l}{n^{2}} \sigma\left(A_{1}\right) q \leq 3 \frac{l}{n^{2}} \sigma(A),
$$


implying

and

$$
\begin{aligned}
(y+l) q & \leq\left(\lambda_{1} \sigma\left(A_{1}\right)+l+1\right) q \leq 280 \frac{l}{q n_{1}^{2}} \sigma(A)+2 l q \\
& \leq 200 \frac{l}{n^{2}} \sigma(A)+6 \frac{l}{n^{2}} \sigma(A) \leq \lambda \sigma(A)
\end{aligned}
$$

$$
\begin{aligned}
z q & \geq\left(\left(1-\lambda_{1}\right) \sigma\left(A_{1}\right)-1\right) q \geq\left(1-\lambda_{1}\right) \sigma(A)-\sigma\left(A_{0}\right)-q \\
& \geq\left(1-\lambda_{1}\right) \sigma(A)-4 l q \geq\left(1-280 l_{1} / n_{1}^{2}-12 l / n^{2}\right) \sigma(A) \\
& \geq\left(1-212 l / n^{2}\right) \sigma(A)>(1-\lambda) \sigma(A) .
\end{aligned}
$$

8. Proof of Corollary 1. We can assume that $q<2 l / n$ with the property that $N_{q}(A)<q-1$ do exist, and let $d$ denote the maximal such $q$. Furthermore, let $A_{0}$ be the set of all elements of $A$ not divisible by $d$ (so that $\left.\left|A_{0}\right|=N_{d}(A)<d-1\right)$, and let $A_{1}$ be the set of integers such that $A \backslash A_{0}=\left\{a d: a \in A_{1}\right\}$; thus, $n_{1}:=\left|A_{1}\right|=\left|A_{d}\right|>n-d$. We have $A_{1} \subseteq\left[1, l_{1}\right]$, where $l_{1}=\lfloor l / d\rfloor$, and we set $\lambda_{1}:=280 l_{1} / n_{1}^{2}$. If $n$ is large enough, then $d<2 l / n<0.1 n$ and

$$
n_{1}>0.9 n \geq 18(l \ln n)^{1 / 2}>20\left(l_{1} \ln n_{1}\right)^{1 / 2} .
$$

As in Proposition 2, maximality of $d$ implies that $N_{q}\left(A_{1}\right) \geq q-1$ for any $q<2 l_{1} / n_{1}$; hence $\left[\lambda_{1} \sigma\left(A_{1}\right),\left(1-\lambda_{1}\right) \sigma\left(A_{1}\right)\right] \subseteq A_{1}^{*}$ by Theorem 1 , and $A_{d}^{*}$ contains all multiples of $d$ from the interval $\left[\lambda_{1} d \sigma\left(A_{1}\right),\left(1-\lambda_{1}\right) d \sigma\left(A_{1}\right)\right]$. It remains to observe that

$$
\lambda_{1} d \sigma\left(A_{1}\right) \leq 280 \frac{l}{d n_{1}^{2}} \sigma(A) \leq 200 \frac{l}{n^{2}} \sigma(A)
$$

and that, in view of $l d<\left(3 l / n^{2}\right) \sigma(A)$ (cf. (13)),

$$
\begin{aligned}
\left(1-\lambda_{1}\right) d \sigma\left(A_{1}\right) & \geq\left(1-\lambda_{1}\right) \sigma(A)-\sigma\left(A_{0}\right) \\
& >\left(1-200 l / n^{2}\right) \sigma(A)-l d>\left(1-203 l / n^{2}\right) \sigma(A) .
\end{aligned}
$$

Appendix. Infinite progressions in subset sums set. Let $\mathcal{A}$ be a (strictly increasing, infinite) sequence of positive integers. Write $\mathcal{A}(n)=$ $|\mathcal{A} \cap[1, n]|$, the counting function of $\mathcal{A}$. A beautiful result of Folkman [Fo66, Theorem 1.3] is that $\mathcal{A}^{*}$ contains an infinite arithmetic progression, provided that $\mathcal{A}(n)>n^{1 / 2+\varepsilon}$ for some fixed $\varepsilon>0$ and all $n$ large enough. In a striking development, Łuczak and Schoen showed recently that the progression can be chosen to be homogeneous and even under a somewhat relaxed assumption on $\mathcal{A}(n)$; their result is, in fact, the infinite version of Theorems $\mathrm{A}$ and B.

TheOREM 5 (Euczak and Schoen, [ŁS00, Theorem 2]). Let $\mathcal{A}$ be a sequence of positive integers satisfying $\mathcal{A}(n) \geq 402 \sqrt{n \ln n}$ for all $n$ large enough. Then there exists an integer $d$ such that $\{d, 2 d, 3 d, \ldots\} \subseteq \mathcal{A}^{*}$. 
In this context we also mention a paper of Hegyvári [H00] where under a similar restriction on $\mathcal{A}(n)$ it is shown that $\mathcal{A}^{*}$ contains an infinite arithmetic progression. However, the progression is not guaranteed to be homogeneous.

An almost immediate consequence of Theorem 5, indicated in [ $\mathrm{ES} 00$ ], is a (nearly sharp) estimate of the maximum possible density of a strongly sum-free sequence of integers.

Theorem 6 (Euczak and Schoen, [ŁS00, Theorem 3]). Let $\mathcal{A}$ be a sequence of positive integers such that the equation $a_{1}+\ldots+a_{k}=a_{0}$ has no solutions in $a_{i} \in \mathcal{A}($ for any $k \geq 2)$. Then $\mathcal{A}(n) \leq 403 \sqrt{n \ln n}$ for all $n$ large enough.

Using Theorem $2^{\prime}$ one can reduce the constants as follows.

THEOREM $5^{\prime}$. Let $\mathcal{A}$ be a sequence of positive integers satisfying $\mathcal{A}(n) \geq$ $5 \sqrt{n \ln n}$ for all $n$ large enough. Then there exists an integer $d$ such that $\{d, 2 d, 3 d, \ldots\} \subseteq \mathcal{A}^{*}$.

THEOREM $6^{\prime}$. Let $\mathcal{A}$ be a sequence of positive integers such that the equation $a_{1}+\ldots+a_{k}=a_{0}$ has no solutions in $a_{i} \in \mathcal{A}($ for any $k \geq 2)$. Then $\mathcal{A}(n)<5 \sqrt{n \ln n}$ for all $n$ large enough.

Theorem $6^{\prime}$ can be deduced from Theorem $5^{\prime}$ exactly in the same way as Theorem 6 is deduced from Theorem 5, and we refer the reader to [ŁS00] for this deduction $\left({ }^{1}\right)$. On the other hand, modifications to be made to the original proof of Theorem 5 (to upgrade it to Theorem $5^{\prime}$ ) are minor but numerous, and for this reason we provide a sketch of the modified proof.

Sketch of the proof of Theorem $5^{\prime}$. Write $\mathcal{A}=\left\{a_{1}, a_{2}, \ldots\right\}$, set $\varphi(i):=$ $\left\lfloor i \ln ^{1 / 4}(i+1)\right\rfloor$ and consider the decomposition $\mathcal{A}=\mathcal{A}_{0} \cup \mathcal{A}_{1}$ defined by

$$
\mathcal{A}_{1}:=\left\{a_{\varphi(1)}, a_{\varphi(2)}, \ldots\right\}, \quad \mathcal{A}_{0}:=\mathcal{A} \backslash \mathcal{A}_{1} .
$$

Evidently,

$$
\begin{aligned}
\mathcal{A}_{1}(n) & =\#\{i: \varphi(i) \leq \mathcal{A}(n)\}=\#\left\{i: i \ln ^{1 / 4}(i+1) \leq \mathcal{A}(n)\right\} \\
& =\frac{\mathcal{A}(n)}{\ln ^{1 / 4} \mathcal{A}(n)}(1+o(1))
\end{aligned}
$$

whence for $n$ large enough we have $\mathcal{A}_{1}(n) \gg \sqrt{n} \ln ^{1 / 4} n$ and $\mathcal{A}_{0}(n)=\mathcal{A}(n)-$ $\mathcal{A}_{1}(n) \geq 4.99 \sqrt{n \ln n}$.

From [Fo66, Lemma 2.2] it follows that the condition

$$
\limsup _{n \rightarrow \infty} \mathcal{A}_{1}(n) / \sqrt{n}=\infty
$$

$\left({ }^{1}\right)$ We have simplified the logic for the sake of clarity. In fact, to deduce Theorem $6^{\prime}$ one needs a version of Theorem $5^{\prime}$ with a constant strictly less than 5 . The reader will see, however, that the argument we present below allows one to obtain any constant greater than $7 / \sqrt{2}=4.949 \ldots$ 
guarantees that for any fixed integer $d \geq 1$ the subset sum set $\mathcal{A}_{1}^{*}$ has bounded gaps on the sequence of all multiples of $d$. That is, to any $d$ there corresponds a constant $C_{d}$ such that $\left\{(y+1) d,(y+2) d, \ldots,\left(y+C_{d}\right) d\right\} \cap$ $\mathcal{A}_{1}^{*} \neq \emptyset$ for any integer $y$. On the other hand, we show that the condition $\mathcal{A}_{0}(n) \geq 4.99 \sqrt{n \ln n}$ ensures the existence of $d$ such that $\mathcal{A}_{0}^{*}$ contains a homogeneous progression with difference $d$ of any pre-assigned length. This will imply that $\mathcal{A}^{*}=\mathcal{A}_{0}^{*}+\mathcal{A}_{1}^{*}$ contains an infinite progression of the form $\{(m+1) d,(m+2) d, \ldots\}$ and therefore the infinite progression $\left\{d^{\prime}, 2 d^{\prime}, \ldots\right\}$ where $d^{\prime}=(m+1) d$.

We need a lemma which is, essentially, [ŁS00, Fact 5].

LEMMA 7. Let $P_{1}$ and $P_{2}$ be homogeneous arithmetic progressions with differences $d_{1}$ and $d_{2}$ and at least $m_{1}$ and $m_{2}$ terms, respectively. Suppose that $m_{2} \geq d_{1}, m_{1} \geq d_{2}$, and $d_{1} \leq d_{2}$. Then $P_{1}+P_{2}$ contains a homogeneous arithmetic progression of difference $d_{1}$ with at least $m_{1}+m_{2}-2 d_{2}$ terms.

For the proof observe that $P_{2}$ contains a homogeneous progression $P_{2}^{\prime}$ of difference $d_{1} d_{2}$ with $\left\lfloor m_{2} / d_{1}\right\rfloor \geq 1$ terms, and the sum of $P_{1}$ and $P_{2}^{\prime}$ is a homogeneous progression of difference $d_{1}$ with at least $\left(\left\lfloor m_{2} / d_{1}\right\rfloor-1\right) d_{2}+$ $m_{1} \geq m_{1}+m_{2}-2 d_{2}$ terms. (We use here the observation that the difference between consecutive terms of $P_{2}^{\prime}$ does not exceed the "length" of $P_{1}$.)

Back to the proof of Theorem $5^{\prime}$, for $i=0,1, \ldots$ we define $l_{i}:=2^{2^{i}}$ (so that $\left.l_{i+1}=l_{i}^{2}\right)$ and we set $A_{i}:=\mathcal{A}_{0} \cap\left(l_{i-1}, l_{i}\right], n_{i}:=\left|A_{i}\right|$. For $i$ large enough we then have $A_{i} \subseteq\left[1, l_{i}\right]$ and

$$
\begin{gathered}
n_{i}=\mathcal{A}_{0}\left(l_{i}\right)-\mathcal{A}_{0}\left(l_{i-1}\right) \geq 4.99 \sqrt{l_{i} \ln l_{i}}-\sqrt{l_{i}} \geq 4.98 \sqrt{l_{i} \ln l_{i}}, \\
\frac{n_{i}^{2}}{49 \ln n_{i}} \geq \frac{4.98^{2} l_{i} \ln l_{i}}{49\left(\ln l_{i}\right) / 2}(1+o(1)) \geq 1.01(1+o(1)) l_{i}>l_{i}, \\
n_{i}>7\left(l_{i} \ln n_{i}\right)^{1 / 2} .
\end{gathered}
$$

Thus Theorem $2^{\prime}$ is applicable and $A_{i}^{*}$ contains a homogeneous progression of difference $d_{i}<5 l_{i} / n_{i}<2 \sqrt{l_{i} / \ln l_{i}}$ and with at least $n_{i}^{2} / 405>\left(l_{i} \ln l_{i}\right) / 20>$ $4 l_{i}$ terms.

Fix an integer $j$ such that the above conclusions hold true and moreover, $d_{i} \geq d_{j}$ for $i \geq j$. Using induction and Lemma 7 it is easy to verify that for any $i \geq j$ the set $A_{j}^{*}+\ldots+A_{i}^{*}$ contains a homogeneous progression of difference $d_{j}$ with at least $4 l_{i}$ terms: put $P_{1}=A_{j}^{*}+\ldots+A_{i-1}^{*}, P_{2}=A_{i}^{*}$ and observe that

$$
\begin{gathered}
d_{i}<2 \frac{l_{i-1}}{\sqrt{2^{i} \ln 2}}<2 l_{i-1}, \quad d_{j}<2 \sqrt{l_{j} /(2 \ln 2)}<2 l_{i}, \\
4 l_{i-1}+4 l_{i}-2 d_{i}=4 l_{i}+2\left(2 l_{i-1}-d_{i}\right)>4 l_{i} .
\end{gathered}
$$

Therefore $\mathcal{A}_{0}^{*}$ contains arbitrarily long homogeneous progressions of difference $d=d_{j}$, as required. 


\section{References}

[AF88] N. Alon and G. A. Freiman, On sums of subsets of a set of integers, Combinatorica 8 (1988), 297-306.

[C90] M. Chaimovich, Subset-sums problems with different summands: computation, Discrete Math. Appl. 27 (1990), 277-282.

[C99] -, New algorithm for dense subset-sum problem, Astérisque 258 (1999), 363373.

[EF90] P. Erdős and G. Freiman, On two additive problems, J. Number Theory 34 (1990), 1-12.

[Fo66] J. Folkman, On the representation of integers as sums of distinct terms from a fixed sequence, Canad. J. Math. 18 (1966), 643-655.

[Fr93] G. A. Freiman, New analytical results in subset-sum problem, Discrete Math. 114 (1993), 205-217.

[GM91] Z. Galil and O. Margalit, An almost linear-time algorithm for the dense subset sum problem, SIAM J. Comput. 20 (1991), 1157-1189.

[H00] N. Hegyvári, On the representation of integers as sums of distinct terms of a fixed set, Acta Arith. 92 (2000), 99-104.

[JŁR] S. Janson, T. Łuczak and A. Ruciński, Random Graphs, Wiley, New York, 2000.

[Le97] V. Lev, Optimal representations by sumsets and subset sums, J. Number Theory 62 (1997), 127-143.

[Le98] -, On consecutive subset sums, Discrete Math. 187 (1998), 151-160.

[Li89] E. Lipkin, On representation of $r$-th powers by subset sums, Acta Arith. 52 (1989), 353-366.

[ŁS00] T. Łuczak and T. Schoen, On the maximal density of sum-free sets, ibid. 95 (2000), 225-229.

[P74] J. M. Pollard, A generalization of the theorem of Cauchy and Davenport, J. London Math. Soc. (2) 8 (1974), 460-462.

[S89] A. Sárközy, Finite addition theorems, I, J. Number Theory 32 (1989), 114-130.

[S94] - Finite addition theorems, II, ibid. 48 (1994), 197-218.

Department of Mathematics

Haifa University at Oranim

Tivon 36006, Israel

E-mail: seva@math.haifa.ac.il

Received on 21.5.2001

and in revised form on 31.3.2002 